\title{
Clinical Application of Ultrasound in the Diagnosis and Treatment of Reninoma
}

\author{
Qiuyang Li, MD ${ }^{a, 1}$, Ying Zhang, MD ${ }^{a, 1}$, Yong Song, MD ${ }^{b}$, Aitao Guo, MD ${ }^{c}$, Nan Li, BS ${ }^{a}$, Yukun Luo, MD ${ }^{a}$, \\ Jie Tang, MD ${ }^{a, *}$ \\ ${ }^{a}$ Department of Ultrasound, First Medical Center of Chinese PLA General Hospital, Beijing, China; ${ }^{b}$ Department of Urology, First \\ Medical Center of Chinese PLA General Hospital, Beijing, China; ${ }^{c}$ Department of Pathology, First Medical Center of Chinese PLA \\ General Hospital, Beijing, China
}

Received March 7, 2020; revision received April 14; accepted May 11, 2020.

Objective: To investigate the clinical value of ultrasound in the diagnosis and treatment of reninoma.

\begin{abstract}
Methods: We retrospectively analyzed the ultrasound findings of 9 patients with reninoma confirmed by pathology after surgical resection in our hospital between September 2012 and August 2019. All patients underwent conventional preoperative ultrasonography. Three underwent contrast-enhanced ultrasound (CEUS) and 3 with complete endogenetic tumor underwent intraoperative ultrasonography.
\end{abstract}

Results: Of the 9 patients with conventional ultrasound, 7 had renal space-occupying lesions and 2 had missed diagnosis. A hypoechoic or hyperechoic solid mass with regular morphology, clear boundary, capsule, weak echo halo around the mass, incomplete thin strip color blood flow signal around the mass were shown in 7 cases. Color Doppler displayed color flow signal of the incomplete thin strip around the mass and arterial blood supply with an internal thin branch. In 3 patients (including 2 with missing diagnosis by conventional ultrasound) who underwent preoperative contrast-enhanced ultrasound, the reninoma manifested as low enhancement, and the perfusion pattern showed as slow wash-in and slow wash-out compared with normal renal cortex. In 3 patients (including 2 cases of missed diagnosis by conventional ultrasound) with completely endogenic reninoma, intraoperative ultrasound clearly showed the tumor characteristics, and all successfully underwent laparoscopic ultrasound-guided partial nephrectomy.

Conclusions: Preoperative conventional ultrasound combined with CEUS, and clinical features, are helpful for the qualitative diagnosis of reninoma. Laparoscopic partial resection is the first-choice treatment for reninoma. Intraoperative ultrasound can provide real-time imaging, accurately evaluate the tumor status, and provide important information for surgeons.

Key words: Reninoma; Contrast-enhanced ultrasound; Intraoperative ultrasound

Advanced Ultrasound in Diagnosis and Therapy 2020;03:211-216

DOI: $10.37015 / A U D T .2020 .200013$

$\mathrm{R}$ eninoma is a rare benign tumor of the kidney. Its tumor cells can secrete a large amount of renin. It originates from the periglomerular cells of the capillaries in the paraglomerular complex, and is also known as juxtaglomerular cell tumor [1]. It was first reported by Robertson et al. In 1967, and the second case by Kihara in 1968, so it is also called Robertson-Kihara syndrome [2]. The clinical manifestations of reninoma include hypertension, hypokalemia, high renin activity and secondary aldosteronism, but the clinical symptoms

\footnotetext{
${ }^{1}$ Qiuyang Li and Ying Zhang contributed equally to this study

* Corresponding Author: Jie Tang, Department of Ultrasound, First Medical Center of Chinese PLA General Hospital, Fuxing Road 28, Beijing, China.

e-mail: txiner@vip.sina.com
} unrestricted use, distribution and reproduction in any medium provided that the original work is properly attributed. 
of some patients are atypical; sometimes because the reninoma is not found in time, long-term hypertension leads to serious damage to important organs, and is even life-threatening. Correct preoperative diagnosis of reninoma is of clinical importance. In the past, there were few cases of reninoma [3], and the imaging manifestations were mainly by computed tomography (CT) and magnetic resonance imaging (MRI) [4-6]. However, there are few reports about its ultrasound manifestations.

Most reninomas are benign. Early detection and surgical resection can achieve good prognosis, and prevent the occurrence of serious complications. The main treatment for reninoma is surgical resection. Radical nephrectomy or simple tumor resection can be used [7]. With the development of laparoscopic technology, laparoscopic partial nephrectomy (LPN) has gradually become routine [8]. Most reninomas are endogenic, central renal tumors with no obvious external processes on the surface of the kidney. It is difficult to identify, locate and remove them under laparoscopy. Removal of too much renal tissue may result in the loss of many nephrons and damage to the integrated system, even leading to urinary fistula and extravasation. In contrast, removal of too little renal tissue may result in tumor residue and positive incisional margins. During laparoscopic surgery, ultrasound can aid by providing a visualize the size, location, shape and blood supply of the tumor.

Reninoma is one of the causes of secondary hypertension. Although it accounts for a small proportion, the incidence is higher in teenagers, and it can be cured by surgery. It is of importance to improve the diagnosis and treatment of this disease. The purpose of this study was to explore the clinical value of ultrasound in the diagnosis and treatment of reninoma.

\section{Materials and Methods}

\section{Ethics approval}

This retrospective study was approved by the Ethics Committee of the Chinese People's Liberation Army (PLA) General Hospital. Written informed consent was obtained from each patient who underwent contrastenhanced ultrasonography (CEUS). All patients agreed to the use of their imaging studies for medical analysis.

\section{Patients}

Nine cases of reninoma were confirmed postoperatively by histopathology between September 2012 and August 2019 in our hospital. These 9 patients (6 male and 3 female, age range $15-30$ years, mean age $23.4 \pm 5.5$ years) underwent conventional ultrasonography,
3 underwent CEUS, and 3 underwent intraoperative ultrasonography. All patients had different degrees of persistent hypertension, headache, dizziness, weakness and blurred vision.

\section{Instruments and methods}

Preoperative conventional ultrasound and CEUS were performed using a Mindray Resona 7, and SC6$1 \mathrm{U}$ abdominal convex array probe, at a frequency of $1-6$ MHz. Intraoperative ultrasound was performed using a BK-2202, equipped with a B-K8826 laparoscopies probe, at a frequency of 4-12 MHz. Contrast agent SonoVue (Bracco, Milan, Italy) was used. Before use, the lyophilized powder of contrast agent was dissolved in $5 \mathrm{ml} 0.9 \%$ sodium chloride solution. It was sufficiently shaken and mixed to form a milky white liquid and extracted, and $0.5 \mathrm{ml}$ was used for each intravenous injection, followed by $5 \mathrm{ml} 0.9 \%$ sodium chloride solution.

After the lesions were displayed by conventional ultrasound and the location, echo, size, shape, boundary and color blood flow were recorded. The best section for displaying the lesions and normal renal parenchyma simultaneously was selected and switched to the contrast mode, and observed for $3 \mathrm{~min}$. The whole process of the examination was recorded and stored in a disk. During the operation, the probe was moved to the tumor location along the surface of the kidney to identify the tumor. After the tumor location and boundary were determined, a mark was cauterized along the edge of the tumor with an electric hook. The tumor and surrounding renal tissue were removed along the mark approximately $0.5 \mathrm{~cm}$ from the edge of the tumor.

\section{Image analysis}

Image analysis was performed by two ultrasound physcians who had $>3$ years experience in abdominal ultrasound and CEUS, and a consensus was reached through discussion. The main outcome measures included tumor echo, size, boundary, pseudocapsule, calcification, cystic change and blood flow signal, and CEUS features.

\section{Results}

All the patients were treated by surgery. Eight patients underwent retroperitoneal laparoscopies nephron-sparing nephrectomy, and 1 patient underwent retroperitoneal laparoscopic radical nephrectomy (Table 1). Diagnosis of reninoma was confirmed by postoperative pathology and immunochemistry All masses were solid and had complete capsules. Histologically, the tumor consisted of ovoid or polygonal cells with a granular, eosinophilic cytoplasm arranged in sheets and trabeculae. All patients 
were positive for renin, CD34 and vimentin. The patient's blood pressure gradually returned to normal within 1-2 weeks after the operation, and no recurrence occurred during the follow-up of 6-72 months.

Table 1 Clinical data and ultrasound features in patients with reninoma

\begin{tabular}{|c|c|c|c|}
\hline Case No. & $\begin{array}{l}\text { Sex, Age (yr), Hypertension } \\
\text { (mm Hg), Clinical symptoms }\end{array}$ & $\begin{array}{l}\text { Tumor location } \\
\text { Tumor size }\end{array}$ & Ultrasound, CEUS, Intraoperative ultrasound \\
\hline 1 & F, 27, 200/150 (mm Hg), Dizziness & $\begin{array}{l}\text { Right/upper } \\
2.2 \times 2.2 \times 1.5 \mathrm{~cm}\end{array}$ & $\begin{array}{l}\text { Ultrasound: Hypoechoic nodule, clear boundary, regular shape, uniform } \\
\text { internal echoes, Color Doppler flow Imaging showed blood flow signal. }\end{array}$ \\
\hline 2 & $\begin{array}{l}\text { M, 23, } 180 / 120, \text { Nausea and } \\
\text { vomiting; chest distress; the whole } \\
\text { body numb and convulsion }\end{array}$ & $\begin{array}{l}\text { Right } / \text { middle } \\
\text { (Complete } \\
\text { endophytic) } \\
3.3 \times 2.5 \times 1.2 \mathrm{~cm}\end{array}$ & $\begin{array}{l}\text { Ultrasound: Hypoechoic nodule with clear boundaries, regular shape and } \\
\text { even internal echo. Color Doppler Flow Imaging showed blood flow signal. } \\
\text { Intraoperative ultrasound: The tumor showed low echo, clear boundary, } \\
\text { regular shape, uneven internal echoes, low echo halo around the mass and } \\
\text { blood flow signal around and inside the tumor in color Doppler. }\end{array}$ \\
\hline 3 & $\begin{array}{l}\text { M, 24, } 170 / 100, \text { Aortic valve } \\
\text { stenosis }\end{array}$ & $\begin{array}{l}\text { Right } / \text { lower } \\
3.5 \times 2.5 \times 2.3 \mathrm{~cm}\end{array}$ & $\begin{array}{l}\text { Ultrasound: Slightly hyperechoic nodule with clear boundaries and regular } \\
\text { shape, Color Doppler Flow Imaging showed no blood flow signal }\end{array}$ \\
\hline 4 & $\begin{array}{l}\mathrm{M}, 19,220 / 110, \text { C e re bra } 1 \\
\text { hemorrhage; secondary epilepsy }\end{array}$ & $\begin{array}{l}\text { Right/upper } \\
\text { (Complete } \\
\text { endophytic) } \\
3.0 \times 2.0 \times 1.0 \mathrm{~cm}\end{array}$ & $\begin{array}{l}\text { Ultrasound: Invisible. } \\
\text { CEUS: Enhancement was gradually increased in the cortical phase, and } \\
\text { gradually increased in the renal parenchymal phase and then decreased } \\
\text { slowly. However, the enhancement degree was always lower than that of the } \\
\text { surrounding normal renal parenchyma, showing low enhancement, and a } \\
\text { perfusion pattern of slow wash-in and slow wash-out. } \\
\text { Intraoperative ultrasound: The tumor showed low echo, clear boundary, } \\
\text { regular shape, uneven internal echoes, low echo halo around the tumor, and } \\
\text { blood flow signal around and inside the tumor at color Doppler. }\end{array}$ \\
\hline 5 & F, 17, 190/140, None & $\begin{array}{l}\text { Right } / \text { middle } \\
2.1 \times 2.0 \times 1.5 \mathrm{~cm}\end{array}$ & $\begin{array}{l}\text { Ultrasound: Slightly hyperechoic nodule with clear boundary and regular } \\
\text { shape, and no blood flow signal in Color Doppler Flow Imaging }\end{array}$ \\
\hline 6 & M, 31, 210/130, None & $\begin{array}{l}\text { Left/upper } \\
2.0 \times 1.5 \times 1.5 \mathrm{~cm}\end{array}$ & $\begin{array}{l}\text { Ultrasound: Hypoechoic nodule, clear boundary, regular shape, and no } \\
\text { blood flow signal in Color Doppler Flow Imaging. }\end{array}$ \\
\hline 7 & $\begin{array}{l}\text { M, 29, 240/140, Functional artery } \\
\text { stenosis; grade III hypertension } \\
\text { fundus change }\end{array}$ & $\begin{array}{c}\text { Left/upper } \\
4.5 \times 3.0 \times 3.0 \mathrm{~cm}\end{array}$ & $\begin{array}{l}\text { Ultrasound: Hypoechoic nodule, with clear boundaries and regular shape, } \\
\text { a mass protrudes from the kidney surface, and Color Doppler Flow Imaging } \\
\text { showed no blood flow signal. }\end{array}$ \\
\hline 8 & F, 26, 120/80, None & $\begin{array}{c}\text { Right/upper } \\
5.0 \times 4.0 \times 3.0 \mathrm{~cm}\end{array}$ & $\begin{array}{l}\text { Ultrasound: Slightly hyperechoic nodule, with clear boundaries and regular } \\
\text { shape, a mass protruding from the kidney surface, and Color Doppler Flow } \\
\text { Imaging showed no blood flow signal. } \\
\text { CEUS: Enhancement was gradually increased in the cortical phase, and } \\
\text { gradually increased in the renal parenchymal phase and then decreased } \\
\text { slowly. However, the enhancement degree was always lower than that of the } \\
\text { surrounding normal renal parenchyma, showing low enhancement, and a } \\
\text { perfusion pattern of slow wash-in and slow Wash-out. }\end{array}$ \\
\hline 9 & M, 15, 190/140, Headache & $\begin{array}{l}\text { Left/upper (Complete } \\
\text { endophytic) } \\
1.0 \times 1.0 \times 0.7 \mathrm{~cm}\end{array}$ & $\begin{array}{l}\text { Ultrasound: Invisible. } \\
\text { CEUS: The enhancement degree was always lower than that of the } \\
\text { surrounding normal renal parenchyma, showing low enhancement, and the } \\
\text { perfusion mode was slow wash-in and slow wash-out. } \\
\text { Intraoperative ultrasound: The tumor showed low echo, clear boundary, } \\
\text { regular shape, uneven internal echoes, low echo halo around the tumor, and } \\
\text { blood flow signal around and inside the tumor on color Doppler. }\end{array}$ \\
\hline
\end{tabular}

Routine ultrasound was performed in 9 patients preoperatively, suggesting renal space-occupying lesions in 7 patients, and no obvious abnormality in 2 . Ultrasound displayed a hypoechoic or hyperechoic solid mass with regular shapes, clear boundary, capsule, and weak echo halo around the mass in 7 cases undergoing conventional ultrasound. The mass protruded obviously outside the kidney, with a maximum diameter of 1.0-5. 0 $\mathrm{cm}$ in 2 cases. Color Doppler showed an incomplete thin strip of color blood flow signals around the mass and arterial blood supply with an internal thin branch.

CEUS was performed preoperatively in 3 patients, of whom 2 had missed diagnosis. CEUS manifestations were as follows: compared with normal renal cortex, the enhancement mode of reninoma was slower to enhanced in the cortical phase, and the degree of enhancement gradually increased and then decreased in the renal parenchymal and delayed phases, but the 
degree of enhancement was always lower than that of the surrounding normal renal parenchyma. The peak and clearance time was slower than that of the normal renal cortex, and the perfusion pattern was manifested as "slow wash-in and slow wash-out" (Fig. 1).

There were 3 cases of completely endogenic reninoma; 2 of which were missed by conventional ultrasound diagnosis. There was no obvious protrusion on the surface of the kidney. During the operation, ultrasound showed that the tumors were clearly visible, with low echo, clear boundary, regular shape, even internal echo, and low echo halo in the surrounding tissues, and color Doppler showed blood flow signals around or inside the tumor. All three patients successfully underwent LPN under the guidance of laparoscopic ultrasonography (LUS), and the pathological results were negative for surgical margins (Fig. 2)
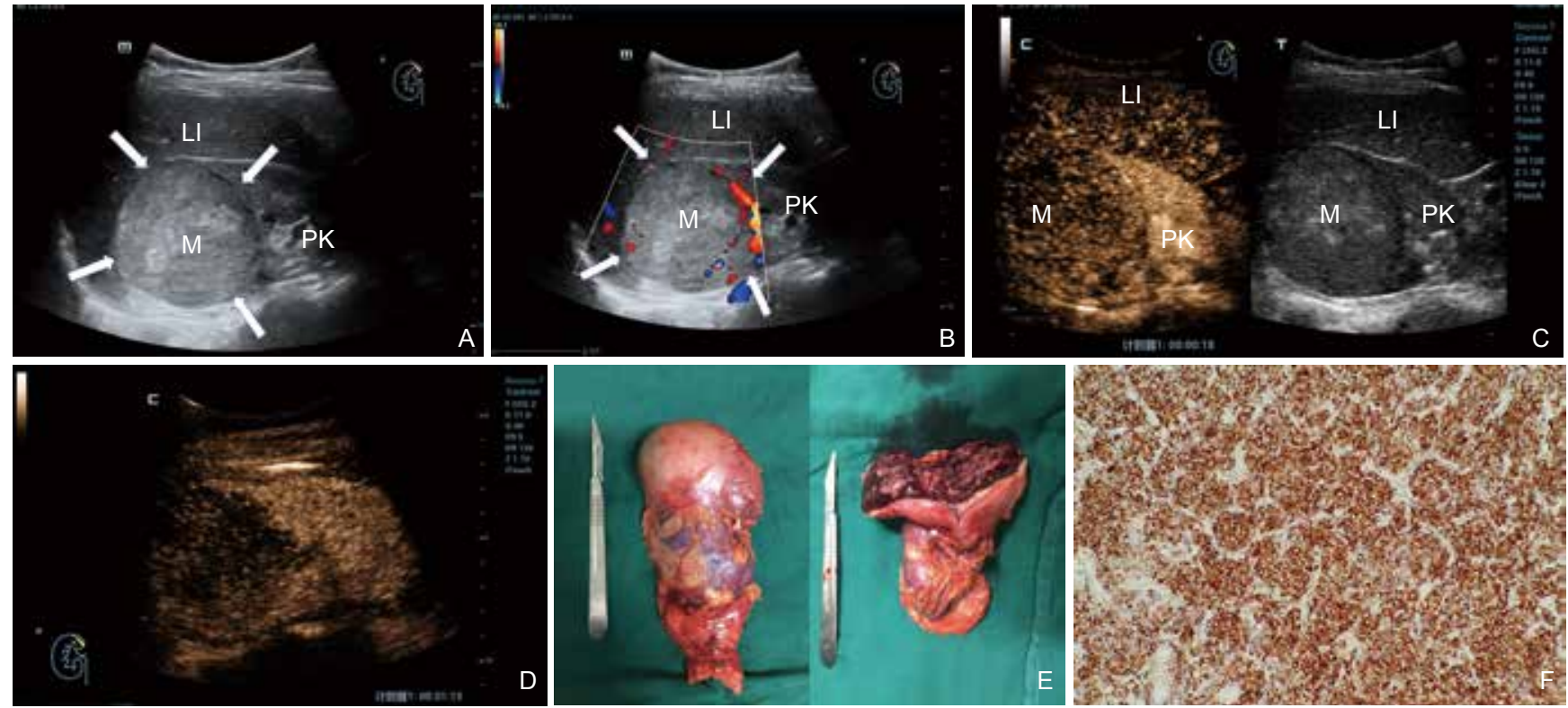

Figure 1 A 26-year-old woman was diagnosed with reninoma on pathological examination. (A) Conventional ultrasound showed a moderate and slightly hyperechoic mass in the upper right kidney (arrow), with clear boundaries and regular shape, protruding from the kidney surface. (B) CDFI showed the blood flow signal (arrow) in and around the mass. (C) CEUS showed that the mass in the cortical phase was slightly enhanced. (D) CEUS showed that the mass in the solid phase was slightly enhanced. (E) Specimen from laparoscopic radical nephrectomy. (F) Postoperative pathology and Immunochemistry suggested reninoma (hematoxylin and eosin, 200').

\section{Discussion}

Reninoma is a rare benign tumor, and the symptoms caused by secondary hypertension can be cured by surgery. Since reninoma was reported for the first time, its origin has been controversial. At present, it is believed to be derived from differentiation of the vascular smooth muscle cells of glomerular arterioles. The paraglomerular cells are located at the glomerular arterioles 2. 0-5. $0 \mu \mathrm{m}$ from the vascular pole of the renal corpuscle. They are mainly derived from the evolved arterial smooth muscle cells. Because they can secrete rennin, they are also known as myoendocrine cells [9]. Reninomas are more common in young people, and most patients are aged 20-30 years. In the present series, the average age of the patients was 23 years, which is similar to that reported in the literature.

Hypertension is the most important symptom of the disease; most of which are serious at $>200 / 120 \mathrm{mmHg}$. The blood pressure may fluctuate in the early stage, and then become persistent, and antihypertensive medication is ineffective. Because of the increase in secondary aldosterone and hypokalemia, patients often have accompanying headache, dizziness, weakness of limbs, and polyuria [10]. The plasma renin level is significantly increased in laboratory examinations. Therefore, when the plasma renin level increases, the possibility of reninoma should be considered [11]. Although there are reports of renal vein invasion and lung metastasis 5 years later [12], the vast majority of reninomas are benign, and surgical resection is currently considered standard of care $[13,14]$.

Imaging examination is helpful in the diagnosis and differential diagnosis of reninoma. Some studies have shown that enhanced CT is an effective imaging method for diagnosis of reninoma at present. Plain scanning shows isodense or low-density masses, and no obvious enhancement in the cortical phase of the tumor. The CT value $(\mathrm{Hu})$ of the solid phase is higher than that in the 
cortical phase. Therefore, tumors in the solid and delayed phases are more visible, with a pattern of slow washin and slow wash-out [15]. MRI findings are similar to CT and show a mass with clear boundaries and lack of specificity. T1-weighted imaging shows an equal- signal or low-signal area, while T2-weighted imaging mainly shows high signals. Diffusion-weighted imaging presents as uniform, halo or nodular high signals, and the enhancement is progressive [5].
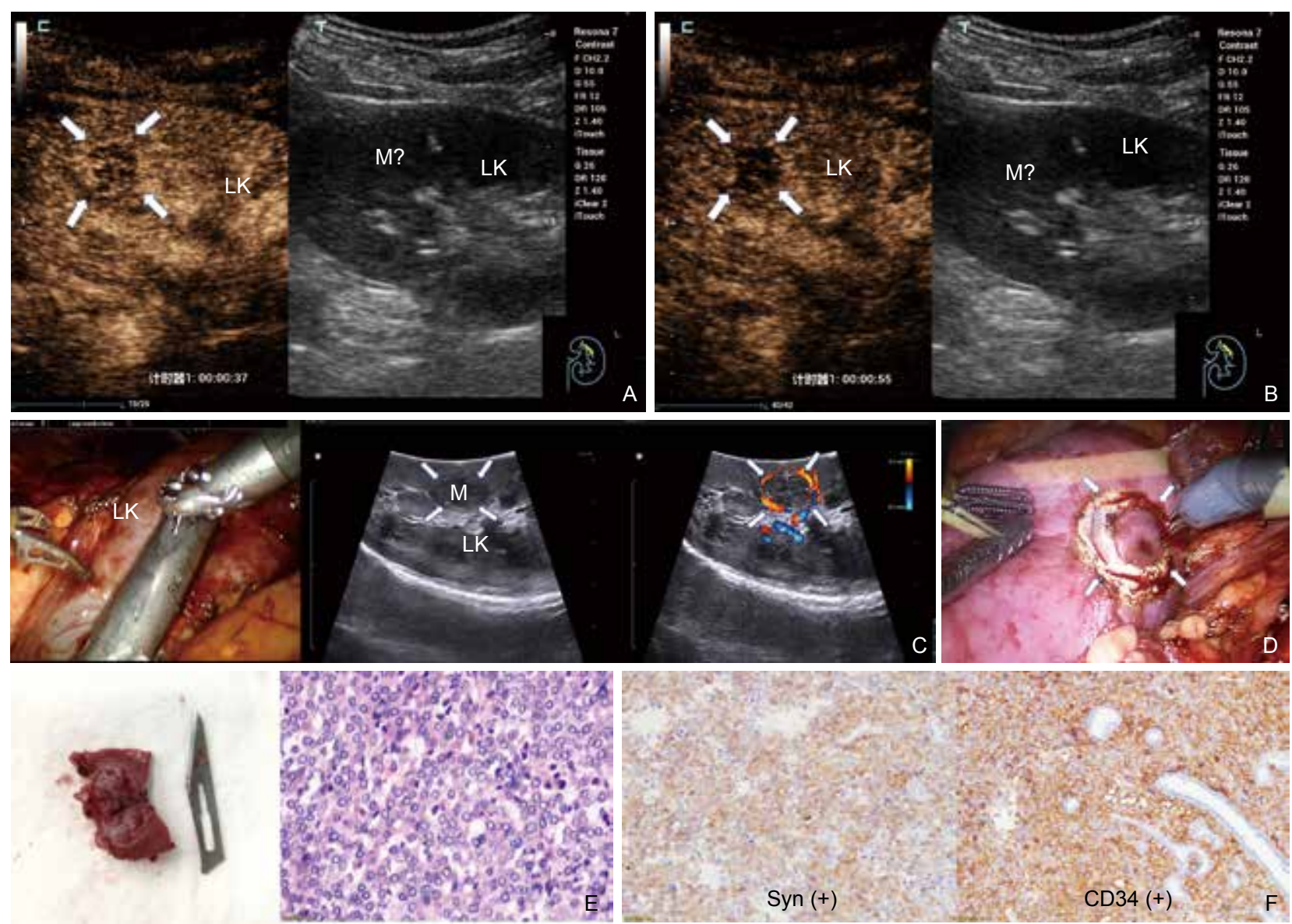

Figure 2 A 15-year-old woman was diagnosed with reninoma on pathological examination. (A) Conventional ultrasound showed left upper renal tumor. CEUS showed a low enhancement focus. (B) CEUS showed continuous low enhancement. (C) Ultrasound showed low echo, clear boundary, regular shape, and low echo halo around the mass, color Doppler showed blood flow signal around and inside the mass. (D) After tumor location and boundary were determined by intraoperative ultrasound, an electric hook was used to mark along the edge of the tumor. (E) Gross tumor specimens were resected along the mark, and sent to pathology, and the pathological findings suggested reninoma (hematoxylin and eosin, 200'). (F) Diagnosis of reninoma was confirmed by immunohistochemistry, Syn (+), CD34 (+) (hematoxylin and eosin, 200).

There are few reports on the diagnosis of reninoma by ultrasound. The present study showed that 7 cases of reninoma manifested as a moderate or slightly high echogenic mass with uniform echo in the renal parenchyma, with clear boundaries, regular shape, capsule, and low echo halo around the mass. Color Doppler showed incomplete a thin strip of color blood flow around the mass and arterial blood supply with an internal thin branch. Conventional ultrasound diagnosis of reninoma has a lack of specific sonographic manifestations, and the tumor is generally small and easy to miss by simply relying on conventional ultrasound. In 2 cases in the present study, conventional ultrasound did not show the presence of the tumor. CEUS is a real-time dynamic imaging method that shows the whole process of contrast agent entering the renal tumor and renal parenchyma until wash-out. In addition, CEUS permits the observation of tumor vascular perfusion in real time. CEUS is better than enhanced CT for distinguishing renal cysts and solid tumors $[16,17]$. In the present study, three patients received preoperative CEUS, which showed slow progression and low enhancement, which was consistent with the contrast-enhanced CT. CEUS succeeded in tumor visualization in two patients whose diagnosis was missed by conventional ultrasound. The reasons for the low enhancement of reninoma imaging are as follows. Although reninoma is rich in thin-walled blood vessels pathologically, and is a vascular-rich 
tumor, renin causes vasoconstriction, and blood vessels are narrowed due to vasoconstriction induced by renin and proliferation of the intima and media of arterioles, finally resulting in decreased blood flow [18].

In recent years, LPN has become the first choice of treatment for reninoma because the tumors are small, common in adolescents, and mostly benign. However, because most reninomas are located in the renal parenchyma, in 3 cases in our study, the tumors were completely endogenic and did not protrude from the surface of the kidney, so it was difficult to determine the location of the tumors during the operation. Under laparoscopy, the ultrasound probe can be placed in the endoscopic channel and performed on the renal surface intraoperatively to determine the tumor size, location, range, texture and blood supply, thus shortening the operation time. In our study, endogenic tumors were located in 3 cases by intraoperative laparoscopic ultrasound, which quickly and accurately determined the location of the tumor, ensured the negative margin, strictly controlled the time of intraoperative ischemia, and attempted to protect renal function.

In summary, reninoma is a rare benign tumor, mostly a single solid mass, which is one of the main causes of secondary hypertension in adolescents. It has characteristic clinical manifestations. Preoperative conventional ultrasound, combined with CEUS and clinical characteristics may be helpful for qualitative diagnosis of reninoma. Laparoscopic partial resection is the first choice of treatment for reninoma. Intraoperative ultrasound can provide real-time images, accurately evaluate tumors, and provide important information for surgeons.

\section{Acknowledgements}

This study was supported by the clinical research fund of PLA General Hospital (No. 2018XXFC-18) and Medical big data research and development project of PLA General Hospital (No. 2019XXMBD-002)

\section{Conflict of Interests}

The authors have no conflict of interest to declare. The funders had no role in study design, data collection and analysis, decision to publish, or preparation of the manuscript.

\section{References}

[1] Vidal-Petiot E, Bens M, Choudat L, Fernandez P, Rouzet F, Hermieu
JF, et al. A case report of reninoma: Radiological and pathological features of the tumour and characterization of tumour-derived juxtaglomerular cells in culture. J Hypertens 2015; 33: 1709-15.

[2] Robertson P. Hypertension due to a renin-secreting renal tumour. American Journal of Medicine 1967; 43:

[3] Oei L, van den Meiracker AH, Oei EH, Moelker A, van Leenders GJ, Rikken B, et al. Reninoma: A rare cause of curable hypertension and hypokalemia. Am J Med 2016; 129: e131-2.

[4] Tanabe A, Naruse M, Ogawa T, Ito F, Takagi S, Takano K, et al. Dynamic computer tomography is useful in the differential diagnosis of juxtaglomerular cell tumor and renal cell carcinoma. Hypertens Res 2001; 24: 331-6.

[5] Kang S, Guo A, Wang H, Ma L, Xie Z, Li J, et al. Magnetic resonance imaging features of a juxtaglomerular cell tumor. $J$ Clin Imaging Sci 2015; 5: 68.

[6] Faucon AL, Bourillon C, Grataloup C, Baron S, Bernadet-Monrozies $\mathrm{P}$, Vidal-Petiot E, et al. Usefulness of magnetic resonance imaging in the diagnosis of juxtaglomerular cell tumors: A report of 10 cases and review of the literature. Am J Kidney Dis 2019; 73: 566-71.

[7] Liu K, Wang B, Ma X, Li H, Zhang Y, Li J, et al. Minimally invasive surgery-based multidisciplinary clinical management of reninoma: A single-center study. Med Sci Monit 2019; 25: 1600-10.

[8] Chen Z, Tang ZY, Liu HT, Chen X. Treatment of juxtaglomerular cell tumor of the kidney by retroperitoneal laparoscopic partial nephrectomy. Urol J 2014; 10: 1160-1.

[9] Barajas L. Anatomy of the juxtaglomerular apparatus. Am J Physiol 1979; 237: F333-43.

[10] Markey RB, MacLennan GT. Juxtaglomerular cell tumor of the kidney. J Urol 2006; 175: 730.

[11] Nunes I, Santos T, Tavares J, Correia L, Coutinho J, Nogueira JMB et al. Secondary hypertension due to a juxtaglomerular cell tumor. $J$ Am Soc Hypertens 2018; 12: 637-40.

[12] Duan X, Bruneval P, Hammadeh R, Fresco R, Eble JN, Clark JI, et al. Metastatic juxtaglomerular cell tumor in a 52-year-old man. Am J Surg Pathol 2004; 28: 1098-102.

[13] MY Mao, B Xia, WL Chen, Song J. Ultrasonic imaging of clear cell sarcoma of kidney: A case report. Advanced Ultrasound in Diagnosis and Therapy 2019; 3: 12-17.

[14] Q Zhang, SP Wei, B Yang, Qian X. Differences between type i and type ii papillary renal cell carcinoma on ultrasound. Advanced Ultrasound in Diagnosis and Therapy 2019; 3: 187-92.

[15] Rosei CA, Giacomelli L, Salvetti M, Paini A, Corbellini C, Tiberio G, et al. Advantages of renin inhibition in a patient with reninoma. Int $J$ Cardiol 2015; 187: 240-2.

[16] Mitterberger M, Pelzer A, Colleselli D, Bartsch G, Strasser H, Pallwein L, et al. Contrast-enhanced ultrasound for diagnosis of prostate cancer and kidney lesions. Eur J Radiol 2007; 64: 231-8.

[17] Aoki S, Hattori R, Yamamoto T, Funahashi Y, Matsukawa Y, Gotoh M, et al. Contrast-enhanced ultrasound using a time-intensity curve for the diagnosis of renal cell carcinoma. BJU Int 2011; 108: 349-54.

[18] Prasad SR, Surabhi VR, Menias CO, Raut AA, Chintapalli KN. Benign renal neoplasms in adults: Cross-sectional imaging findings. AJR Am J Roentgenol 2008; 190: 158-64. 